\title{
Políticas de formación profesional en el área de información en el Mercosur: reflexiones acerca de una experiencia de armonización curricular en Biblioteconomía
}

\author{
Ely Francina Tannuri de Oliveira \\ José Augusto Chaves Guimarães \\ Universidade Estadual Paulista (Marília, Brasil)
}

\subsection{Resumen}

Se analiza la evolución de la formación universitaria del profesional bibliotecario, así como el proceso de armonización curricular en el área de Biblioteconomía a partir de 1996, como elementos para la construcción de una política educativa que pueda conducir a un modelo integrador de educación superior en el marco del Mercosur.

Palabras clave: Biblioteconomía. Formación profesional. Educación. MERCOSUR. Bibliotecarios. Profesionales de información.

\subsection{Abstract}

Considering the creation of Mercosul in 1991 in order to broading the national markets of Argentine, Brazil, Paraguay and Uruguay as well as to guarantee a free flow of goods, services and products, an historical overview the undergraduate library education is provided and the curricular harmonizing process started in 1996 is analyzed. Such aspects furnish background for pointing out some challenges and perspectives for building library education politics in Mercosul which could lead to an integrated higher education model.

Keywords: Mercosul. Library education. Information professionals.

\section{Introducción}

En América Latina, la idea de integración se discute hace mucho tiempo. En ese contexto, el Mercado Común del Sur (Mercosur), como instancia de integración económica entre Argentina, Brasil, Uruguay y Paraguay, tuvo su origen en 1980, cuando se firmaron once acuerdos de cooperación entre Argentina y Brasil. En 1985, Brasil y Argentina firmaron la Declaración de Iguazú, que abrió 
el camino a una serie de tratados en el campo económico y fue una contribución importante a la formación del Mercosur en 1991. Creado el 29 de marzo de 1991, con el objetivo de ampliar las dimensiones de los mercados nacionales de Argentina , Brasil, Paraguay y Uruguay, y de promover la libre circulación de bienes y servicios, y factores de producción en la región, el Mercosur se presentó, sobre todo, como una perspectiva de vida más digna para la población de los países miembros. Según Morosini (1998 a, p. 28), el Mercosur "se constituye en la iniciativa internacional más importante de la última década del Cono Sur y se propone realizar una importante reestructuración política en esos países". Para Lampert (1998, p.2) el Mercosur "es un intento de que los países de América del Sur se organicen, después de largos años de dictadura militar, para enfrentar conjuntamente las constantes crisis políticas, económicas y sociales que afectan a la vida del ciudadano suramericano".

En el contexto del Mercosur, se puede observar un amplio conjunto de acuerdos constituidos entre los estados miembros, en aspectos como la libre circulación de bienes, servicios y factores productivos entre los países, a través de la eliminación de derechos aduaneros y de los impuestos de circulación de mercancías; el establecimiento de un impuesto externo común y la adopción de una política comercial en relación a terceros estados; la coordinación entre los estados miembros de políticas macroeconómicas y sectoriales de comercio exterior, agrícultura, industria, fiscalidad, moneda, cambio y capitales, servicios, aduanas, transportes y comunicaciones. La educación se mostró, desde el primer momento, elemento clave para la integración regional, en la medida en que podría contribuir de forma notable a la superación de las disparidades regionales, la consolidación de la democracia y el desarrollo económico y social. Así, paralelamente a las negociaciones económicas, en la II Reunión del Consejo del Mercado Común realizada en Argentina, el 26 y 27 de junio de 1992 fue creado el Plan Trienal para el Sector Educacional en el Proceso de Integración del Mercosur. Este plan preveía la constitución de una Comisión de Ministros en el área de Trabajo, Justicia y Educación, con la función de proponer al Consejo del Mercado Común medidas con vistas a la coordinación de políticas educativas entre los estados miembros. La Comisión es asesorada por comités regionales, que tienen por objetivo la elaboración de proyectos y programas significativos para el proceso de integración y desarrollo de las diferentes regiones. La internacionalización de los sistemas educativos $-\mathrm{y}$, en este caso específico, la integración universitaria en el Mercosur - se consideran importantes en la medida en que hacen posible su inserción activa en el contexto mundial.

Así, teniendo como presupuesto el nuevo orden mundial marcado por la globalización de los mercados, el agrupamiento de los países en grandes megabloques regionales y la internacionalización de los sistemas de enseñanza, este

Scire. $10: 1$ (en.-jun. 2004) 145-157. 
artículo se propone presentar el panorama de la formación inicial del profesional bibliotecario en los países que constituyen el Mercosur, su articulación en términos de políticas educativas conjuntas, y las tendencias y desafíos en la formación de este profesional que deben ser tenidos en cuenta. En términos metodológicos, se parte de una revisión documental de los aspectos históricos que determinaron el desarrollo de los estudios para, a continuación, analizar la literatura relativa a la armonización curricular en Biblioteconomía en el ámbito del Mercosur.

\section{La formación universitaria bibliotecológica en el Mercosur}

Desde su creación, el Mercosur concedió una gran relevancia a la educación como factor de integración, viendo en ella la pieza clave para la consolidación de la unión que comenzaba a construirse entre los países. Además de eso, se le reconocía la importancia de promover el reencuentro entre los pueblos de la región con respecto a la búsqueda de valores comunes sin, no obstante, menoscabo de sus propias identidades. En diciembre de 1991, por medio de la Resolución 07/ 92, se creó la reunión de los Ministros de Educación de los países miembros del Mercosur, que aprobó los Planes Trienales de Educación, destinados a orientar los trabajos del Mercosur Educativo, y que fueron firmados por los ministros de Argentina, Brasil, Paraguay y Uruguay, países miembros, y de Chile y Bolivia, países asociados. Tales planes se proponen, además de la formación de una conciencia ciudadana favorable al proceso de integración, la capacitación de recursos humanos y la compatibilización y armonización de los sistemas educativos.

En realidad, la cuestión de la internacionalización de la educación superior, como fortalecimiento de la competitividad necesaria para inserirse en el contexto mundial, ya constaba como una de la principales tendencias de la educación superior en América Latina desde la década de los sesenta, que se ha acentuado en las dos últimas décadas. Esto supone modelos educativos de enseñanza superior en los que los currículos se armonicen y la formación profesional se realice a través de la articulación de ejes comunes.

En términos históricos, se observa que, en los diversos países del Mercosur, la formación biblioteconómica ha estado marcada por la fusión de dos corrientes: la humanista, con raíz europea en la École Nationale de Chartres, creada en París en 1821 (pionera en la enseñanza de Biblioteconomía), y la técnica, de raíz norteamericana. Es normal que el primer curso de Biblioteconomía se impartiera en Argentina en 1822, dado el interés por las bibliotecas existente en ese país. Cumple observar que en el siglo XIX, época en la que se crea la Biblioteca Nacional de aquel país, había ya abundante literatura sobre bibliotecas escolares y públicas. A comienzo del siglo XX aparecen las primeras bibliotecas especializadas y universitarias. Como resalta Fernández (1979, p. 7) "en 1910 Buenos Aires por el conjunto de sus bibliotecas era uno de los primeros centros culturales de América Latina".

Scire. $10: 1$ (en.-jun. 2004) 145-157. 
El primer programa sistemático de formación de bibliotecarios en América Latina se inició en Río de Janeiro en 1915, con currículo nítidamente de influencia humanista europea, ya que en Brasil, en junio de 1911, se había creado el "primer curso de Biblioteconomía en la Biblioteca Nacional de Río de Janeiro que comenzó a funcionar efectivamente en 1915, bajo la influencia de la École Nationale de Chartres. En octubre de 1929, se instaló en el Instituto Mackenzie, de origen americano, el segundo curso de Biblioteconomía del país, con orientación típicamente americana. La influencia francesa se deja de lado, para inscribirse en una Biblioteconomía típicamente americana. Ese modelo norteamericano sobrevivió en la formación de bibliotecarios en Brasil hasta la década de los ochenta. De esta forma, el país contó, al final de los cuarenta, con cinco cursos a los cuales se sumaron otros tres en la década de los cincuenta. Resalta, en este contexto, el hecho de que la Biblioteconomía había sido reconocida como profesión liberal en 1959 y como formación de nivel superior en 1962, cuando se instituyó su primer currículo mínimo." (Oliveira, 1996, p. 24).

En 1922 se inicia en Argentina la primera escuela argentina - y la primera a nivel universitario de América Latina - inicia su andadura en la Facultad de Filosofía y Letras. Su propuesta inicial pretendía formar al archivista y al bibliotecario para los servicios de museos, con un currículo de tendencia marcadamente humanista, con asignaturas orientadas a la Literatura y la Filosofía, identificándose con la corriente europea. En 1937 se inicia un segundo curso de Biblioteconomía en la Escuela de Servicio Social, dependiente del Museo Social Argentino, de carácter privado y de inspiración norteamericana. En 1951 se transforma en curso superior. Desde ese momento se suceden eventos y congresos, siempre con la preocupación de la formación profesional y la armonización de sus programas y currículos. Hoy Argentina cuenta con siete carreras universitarias de Bibliotecología, ubicadas en siete provincias del país. Argentina, a diferencia de los demás países del Mercosur, posee tres niveles de formación bibliotecaria: bibliotecario escolar, bibliotecario-documentalista y licenciado en Bibliotecología. La Universidad Nacional de Mar del Plata ofrece los tres.

En Chile, la enseñanza de la Biblioteconomía se inició en 1946, con cursos de pequeña duración. Inicialmente después de transformarse en curso de nivel superior, la carrera de Biblioteconomía estuvo bajo la responsabilidad de la Universidad de Santiago. Chile, aunque no sea integrante oficial del Mercosur, ha participado desde el comienzo de las discusiones curriculares en el área de Biblioteconomía.

En Paraguay, la única Escuela de Biblioteconomía fue creada en 1971, pasando a tener nivel universitario solamente en 1986. Actualmente funciona anexa a la Facultad Politécnica. Según los registros, la existencia de la Escuela de Biblioteconomía se debe, principalmente, al espíritu de lucha de sus profesores, 
que no escatimaron esfuerzos para implementar la profesión bibliotecaria en la sociedad paraguaya, especialmente al principio, entre 1971 y 1978.

En Uruguay, la Escuela Universitaria de Biblioteconomía y Ciencias Afines es, también, la única institución de enseñanza superior que forma licenciados en Biblioteconomía y Archivología, a nivel nacional. Institucionalmente, forma parte de la Universidad de la República Oriental de Uruguay. Se inició de forma incipiente en 1943, con impartiendo cursos de formación de bibliotecarios, desde 1945 se convirtió en un curso universitario, y desde 1959 pasó a depender directamente del Consejo Directivo Central de la Universidad de la República, situación que se mantiene hasta hoy.

En síntesis, la creación de los primeros cursos de Biblioteconomía en los diferentes países del Mercosur se inició en el siglo XIX (Argentina, 1822), tuvo gran preponderancia en la primera mitad del siglo XX (Brasil, 1911; Uruguay, 1943; y Chile, 1946) extendiéndose hasta los años setenta (Paraguay, 1971). A partir de la década de los sesenta, tanto en Argentina como en Brasil, comienzan a multiplicarse los cursos de Biblioteconomía. Entre los factores que influyeron en la enseñanza de la Biblioteconomía al final de la década de los sesenta y comienzo de los setenta, el más relevante fue el desarrollo de la enseñanza superior. Especialmente en Brasil, junto con el desarrollo tecnológico y a los cambios sociales y económicos, este factor influyó fuertemente en los programas de formación profesional en el área, ya que provocó la expansión de los cursos de graduación y la creación de los de postgrado - programas de maestría y doctorado en Biblioteconomía y Ciencia de la Información - , hasta entonces inexistentes y actualmente ocho. Hoy en día, los países del Mercosur cuentan con un conjunto de 46 cursos universitarios de Biblioteconomía: siete en Argentina, treinta y cinco en Brasil, dos en Chile, uno en Paraguay y uno en Uruguay (1).

\section{Armonización curricular: la construcción de un espacio}

Las iniciativas de integración regional en el ámbito de las escuelas de Biblioteconomía del Mercosur se remontan al inicio de la década de los noventa, bajo dos vertientes: por un lado, se registra la iniciativa uruguaya, bajo la coordinación de María Teresa Castilla, relativa a la creación de una asociación de escuelas de Biblioteconomía del Cono Sur, integrando las escuelas argentinas, chilenas, paraguayas y uruguayas (pero sin prever la inclusión de Brasil); y, por otro lado, la iniciativa brasileña, cuando la ya consolidada experiencia de estudios curriculares por regiones del país pudo, por primera vez, ser discutida con los demás países latinoamericanos en el III Seminário Nacional de Avaliação Curricular em Biblioteconomia (Belo Horizonte, 1994). En esa ocasión, fue posible establecer mayores contactos con profesores como Elsa Barber (Argentina), Mariano Maura (Puerto Rico), Gloria Ponjuan (Cuba) y Octavio Castillo Scire. $10: 1$ (en.-jun. 2004) 145-157. 
(Panamá), entre otros. Tales aspectos propiciaron que en 1996, la Associação Brasileira de Escolas de Biblioteconomia e Documentação (ABEBD) pudiese participar en el III Encuentro de Educadores e Investigadores en Bibliotecología Ciencias de la Información y Documentación de Iberoamérica y el Caribe (San Juan, Puerto Rico), donde se discutió una propuesta de áreas curriculares en Biblioteconomía para la región.

Como consecuencia, en el mismo año, fue posible promover en Porto alegre (Brasil), el I Encontro de Dirigentes de Escolas Universitárias de Biblioteconomia do Mercosul, que contó con la participación de representantes de la mayoría de los cursos brasileños, así como de los siete cursos de Argentina, de los dos de Chile y de ambos cursos de Uruguay y de Paraguay. El referido evento propició que, por primera vez, hubiese un espacio de conocimiento mutuo y de intercambio de experiencias entre las escuelas de la región, ocasión en la que los países participantes firmaron un acuerdo refrendando seis de la siete áreas curriculares propuestas en la reunión de Puerto Rico - Fundamentos de Biblioteconomía y Ciencia de la Información, Tratamiento de la Información, Recursos y Servicios de Información, Gestión de la Información y Nuevas Tecnologías e Investigación-, excluyendo sólo el área de Práctica profesional por considerarla un instrumento de carácter transversal. A partir de ese evento, se inició un proyecto de armonización curricular en Biblioteconomía en el Mercosur (Argentina, Brasil, Chile, Paraguay e Uruguay), cuyo espacio natural serían los encuentros que se realizarían anualmente, una vez en cada país.

Para continuar con el proyecto, en noviembre de 1997, se realizó en Buenos Aires el II Encuentro de Directores y I de Docentes de Bibliotecología del Mercosur, ocasión en la que cada área pudo, por medio de talleres de discusión, elaborar enmiendas básicas con los contenidos curriculares fundamentales. Igualmente se discutieron cuestiones relativas a la incentivación de la capacitación docente y a las perspectivas de integración regional en términos de investigación. Al año siguiente (octubre, 1998) se realizó en Santiago de Chile el III Encuentro de Directores y II de Docentes, donde fue posible establecer los objetivos de cada área curricular, y se realizo un análisis del énfasis, en términos cuantitativos, que debería ser dado a cada área respecto a las demás.

La cuestión de los contenidos curriculares en Biblioteconomía en el Mercosur adquirió mayor profundidad cuando, en mayo de 2000, el IV Encuentro de Directores y III de Docentes (Montevideo) propició la discusión del conjunto de marcos teórico- metodológicos de cada una de las seis asignaturas, promoviendo un paso adelante en lo referido al quehacer pedagógico de las escuelas de la región y a la construcción del conocimiento en ellas desarrollada. Igualmente, se definió, en el ámbito del Encuentro de Directores, el conjunto de competencias profesionales a alcanzar por los egresados de los cursos de Biblioteconomía del

Scire. $10: 1$ (en.-jun. 2004) 145-157. 
Mercosur. En este sentido, la comisión inter-países responsable del documento final (2) que fue discutido y aprobado en plenario partió de la identificación de los problemas u obstáculos a los que se debían enfrentar los cursos en el desarrollo de las competencias profesionales, para, inmediatamente después, establecer una categorización de las competencias profesionales (3), conforme a la propuesta de Vera Beraquet, en competencias de comunicación y expresión, técnicocientíficas, gerenciales, y sociales y políticas.

Una vez abordadas las cuestiones de contenido, el grupo dirigió su atención a la operabilidad de esos contenidos en el ámbito de las escuelas: el quehacer pedagógico. De esa forma el $V$ Encuentro de Directores y $I V$ de Docentes de Biblioteconomía del Mercosur, realizado en Asunción (Paraguay), en julio de 2001, abordó el perfil del docente de Biblioteconomía en aspectos como su formación científica y pedagógica, sus prácticas pedagógicas y las políticas institucionales necesarias para impulsar tales aspectos. El evento contó con la participación de Bolivia, como candidata a una posible integración en el grupo. Considerando el hecho de que los cuatro eventos anteriores habían centrado sus esfuerzos en la cuestión curricular, esta vez el foco de la discusión se centró en la figura del docente, entendido como docente-investigador; aspectos que se encontraban expresados en el tema central de ambos eventos -Directrices político-estratégicas con impacto en la investigación y la extensión (Encuentro de Docentes) y El docente de Biblioteconomía en la sociedad del conocimiento (Encuentro de Docentes) - . La reflexión se centró en el perfil y las dimensiones éticas de la responsabilidad social de ese docente en el contexto de los cursos de Biblioteconomía del Mercosur sin perder de vista las innovaciones exigidas por los nuevos tiempos. De esta manera, en el Encuentro de Directores fue posible analizar el " $[\ldots]$ cuadro comparativo trazado sobre el ejercicio docente en los países del Mercosur, en el que se consideraron las semejanzas y diferencias entre los diversos países según los siguientes indicadores: requisitos para ascender a la docencia (concursos, exámenes, etc.), mecanismos de promoción docente, aspectos legales del ejercicio de la docencia, optimación del trabajo docente y evaluación de la actuación docente" (Oliveira, 2001, p.1). De esta forma, como resaltan Guimarães \& Rodrigues (2002), por primera vez en el ámbito de los referidos eventos le fue posible al grupo, "tener una idea que abarcase más la realidad docente de la región, aspecto que permitió la discusión de perspectivas de intercambio y de programas conjuntos futuros". Como tónica general de las discusiones del evento, quedó patente la cuestión de la investigación como una necesidad básica para el docente, incluso como elemento para nutrir su propia práctica pedagógica, incluyéndose aquí aspectos no sólo relativos a la investigación para la enseñanza, sino también a la enseñanza a través de la investigación (4). En las conclusiones del evento quedó claro, para el grupo de escuelas de la región, el

Scire. $10: 1$ (en.-jun. 2004) 145-157. 
corte transversal que la investigación y las nuevas tecnologías desempeñan en el ámbito de la enseñanza de la Biblioteconomía, debiendo, por ello, ser objeto de incorporación a la actividad docente de las distintas áreas temáticas. Así, desde el evento de Asunción, los estudios curriculares de Biblioteconomía del Mercosur pasaron a contar con cuatro áreas temáticas - Fundamentos de Biblioteconomía y Ciencia de la Información, Organización y Tratamiento de la Información, Recursos y Servicios de Información, y Gestión de la Información - y dos áreas instrumentales - Nuevas tecnologías de la información e Investigación-.

Una vez concluida la primera ronda de eventos en cada uno de los cinco países, Brasil acogió nuevamente el VI Encontro de Diretores e V de Docentes de Biblioteconomia e Ciência da Informação do Mercosul (Londrina, del 22 al 25 de octubre de 2002), teniendo como tema la "Investigación el los cursos de Biblioteconomía del Mercosur en sus relaciones con la enseñanza y la extensión universitaria" y como subtemas: investigación docente, investigación discente, líneas y grupos de investigación, formas de apoyo y fomento de la investigación, programas y políticas institucionales de investigación, la investigación en la enseñanza y la enseñanza de la investigación, la investigación y la extensión/servicios a la comunidad, la investigación como forma de integración entre la graduación y el postgrado, la base científica del quehacer académico y las estrategias de efectivización de la investigación como elemento que permea la totalidad del proceso educativo. Entre las recomendaciones del Encuentro de Directores (Encontro de Diretores, 2002, p. 2-3), destaca la preocupación por la divulgación de las actividades de investigación desarrolladas en los distintos cursos de Biblioteconomía de la región por medio de sus respectivas páginas web, más específicamente en lo que toca "a datos curriculares y de producción científica de los docentes -en el caso de Brasil con un enlace con los Currículos Lattes -, líneas de investigación del curso (título y sumario), trabajos de conclusión de curso (tesinas) de los alumnos (datos referenciales y resúmenes)". Así, se sacó a colación por primera vez, en el referido espacio, una cuestión fundamental: la visibilidad científica de los cursos. Se trataba no sólo de favorecer la divulgación de las investigaciones en el ámbito científico del área (5), sino también de aumentar el intercambio investigador entre los cursos, abriendo la posibilidad de formar grupos interinstitucionales sobre temáticas comunes. Para ello, se recomendó "que cada escuela haga un levantamiento de los convenios-marco existentes con otras IES del área del Mercosur y los encamine a la coordinación nacional para su divulgación por la ABECIN y, para que se pueda, en el próximo Encuentro, delinear acciones conjuntas de capacitación e investigación" (6).

Como resultado, otra recomendación del evento señaló la necesidad de investigar las cuestiones educativas específicas de las cuatro áreas curriculares por medio de grupos de estudio. Con el objetivo de sistematizar el conocimiento 
hasta entonces generado en el transcurso de esos seis años del Grupo de Escuelas de Biblioteconomía del Mercosur, se llegó al acuerdo de editar un libro, estructurado en las cuatro áreas curriculares y en las dos instrumentales, de manera que se rescatase críticamente la visión de área. La coordinación de cada área quedó de esta manera: Fundamentos de Biblioteconomía y Ciencia de la Información (Argentina), Organización y Tratamiento de la Información (Brasil), Recursos y Servicios de Información (Uruguay), Gestión de la Información (Chile), Nuevas Tecnologías (Paraguay) e Investigación (Grupo ínter-países bajo la coordinación de la profesora Mara Rodrigues, Brasil). Cabe destacar que el presente estudio fue decidido como modelo metodológico para la estructuración de los capítulos, debiendo su síntesis constituirse en el capítulo referente al área 2, a cargo de Brasil. El Encuentro de Docentes, a su vez, propició la realización de grupos de trabajo en las cuatro áreas curriculares (Encontro de Directores..., 2002, p. 1) con los siguientes objetivos específicos: a) definir estrategias para el desarrollo y consolidación de la investigación docente y discente; b) proponer acciones de creación y consolidación de líneas y grupos de investigación; c) sugerir formas de buscar apoyo para la investigación; d) proponer metodologías para establecer la investigación como principio educativo; e) proponer metodologías para establecer la investigación como forma de integración entre grado y postgrado; y f) definir estrategias para crear una base científica del quehacer académico a partir de la concepción de la investigación como elemento permeador de todo el proceso educativo. De ese modo, tras definir las áreas Investigación y Nuevas Tecnologías como transversales que traspasan las cuatro áreas de contenido, se especificó como tema del VII Encuentro de Directores y VI Docentes de Biblioteconomía del Mercosur, que se realizará en Mar del Plata (Argentina) en agosto de 2004, la discusión de las interfaces de las Nuevas Tecnologías con los quehaceres curriculares específicos, así como las políticas institucionales para concretar el acceso efectivo de las diferentes escuelas a ese ambiente tecnológico.

\section{Consideraciones finales: desafíos y perspectivas}

Cada uno de los países del Mercosur se encuentra en un momento diferente en lo que concierne al desarrollo y a la implementación de políticas para la enseñanza superior, hecho éste que se refleja también en la enseñanza de la Biblioteconomía. Una posible integración requiere minimizar estos puntos de diferencia, no en el sentido de nivelar las características, sino en el sentido de encontrar puntos de identidad entre los diferentes sistemas de educación superior, tanto en lo que concierne a la organización y objetivos de los cursos como en lo relacionado a la propia estructuración curricular. Un punto de encuentro lo constituye sin duda el hecho de que actualmente todos los países compartan los objetivos y los principios de una educación de carácter democrático.

Scire. $10: 1$ (en.-jun. 2004) 145-157. 
A corto plazo, se presentan algunos desafíos para la integración. La primera cuestión está relacionada a la validez de los diplomas universitarios. El desarrollo previsto en el Plan Trienal de Educación de del Mercosur 2000 necesita una consolidación más eficaz. Los protocolos de enseñanza superior acordados desde 1996 - que abordan los estudios de postgrado, la formación de recursos humanos para el postgrado, y el reconocimiento de títulos y grados universitarios - deberán ser transformados en normas jurídicas, para lo que es necesario que los agentes sociales y sindicales de las diferentes áreas de conocimiento tomen una posición, pues la libre circulación de profesionales está íntimamente relacionada al reconocimiento y la convalidación de los diplomas universitarios. Otra cuestión importante es la relativa a los conceptos pedagógicos comunes, los pilares sobre los cuales construir la integración. De hecho, el primer momento de la armonización curricular se produce cuando se hacen propuestas pedagógicas comunes para los cursos de todos los países. Así, por ejemplo, aumentar la investigación en la graduación es una propuesta que ofrece un elemento de intervención en lo cotidiano, garantizando un egresado apto para desafiar circunstancias diversas en el mercado de trabajo, capaz de actuar en diversas instancias y en un universo geográfico más amplio. Otro aspecto que parece fundamental, pensando en la formación del profesional bibliotecario que actuará los próximos años, es la necesidad de la educación continua, que está en proceso de consolidación en todo el Mercosur, especialmente en el ámbito nacional. En definitiva, los encuentros, los congresos, los simposios y cursos realizados por las diferentes instituciones hacen viable la integración, ya que se constituyen en puntos de encuentro en los que los profesionales de las diferentes instituciones y de los diferentes países contemplan temáticas y problemas comunes.

En conclusión, el Mercosur articula la educación en el proceso de integración, delineando la construcción de un nuevo modelo de educación superior: un modelo integrado que transcienda los límites de las naciones, que facilite la movilidad de las personas, que propicie la integración económica política y social, y que contribuya a la formación de profesionales capaces de actuar fuera de los estrechos límites nacionales, comprometidos con la mejora de la calidad de vida de la población. En ese sentido, cabe resaltar el carácter pionero de la experiencia de conocimiento mutuo, intercambio de información y armonización curricular que el grupo de directores y docentes de Biblioteconomía del Mercosur ha realizado desde 1996; experiencia que hace abrigar fundadas esperanzas de que este anhelado modelo de educación superior llegue a alcanzarse.

\section{Notas}

(1) La dirección de las escuelas se puede encontrar en la página de la Associação Brasileira de Ensino de Biblioteconomia e Documentação. 
(2) Integraron la referida comisión los profesores Noemi Conforti (Argentina), Vera Beraquet (Brasil) Christian Valenzuela (Chile), Valeriana Bernal de Vega (Paraguay) y Mário Barité (Uruguay).

(3) El documento (VALENTIM, 2000, p.17) define competencias profesionales como el "conjunto de habilidades, destrezas y conocimientos que debe poseer un profesional en cualquier disciplina, para cumplir con su actividad especializada, ofreciendo un mínimo de garantía de los resultados de su trabajo, tanto a sus clientes o empleadores como, en última instancia, a la sociedad de la cual forma parte".

(4) Rodrigues, Mara Eliane Fonseca, A docência universitária e os desafios do mundo moderno: reflexões sobre a prática pedagógica do docente de Biblioteconomia; Ayala Rodríguez, María Soledad, Incorporación de la investigación en la formación de profesionales; y Escobar de Morel, Margarita, La función docente integrada a la extensión y la investigación.

(5) Se resalta, en este ámbito, la preocupación por la divulgación de la información generada en el espacio del Mercosur; aspecto que se exterioriza en las recomendaciones de transformar el Observatorio Mercosur en una sección de la página web de la ABECIN - alimentada con continuidad por la Universidad de la República (Uruguay) _, de estudiar de cara al próximo evento la creación de un portal para guardar la información del espacio común y, principalmente, la puesta en marcha de una revista científica electrónica dedicada a las cuestiones de enseñanza de Biblioteconomía en el Mercosur.

(6) La ABECIN ( Associação Nacional de Educação em Ciência da Informação), creada en 2001, en sustitución de la Associação Brasileira de Escolas de Biblioteconomia e Documentação, tiene como objetivo las discusiones educativas en el área, en Brasil.

\section{Referencias}

Associação Brasileira de Ensino de Biblioteconomia e Documentação (1998). Moderno profissional da informação: o perfil almejado pelos cursos de Biblioteconomia brasileiros. Porto Alegre: Associação Brasileira de Ensino de Biblioteconomia e Documentação, 1998.

Associação Brasileira de Ensino de Biblioteconomia e Documentação (1996). Encontro dos dirigentes dos cursos superiores de Biblioteconomia do Mercosul: a formação profissional em Biblioteconomia no Mercosul. Porto Alegre, 1996. 2 v.

Associação Brasileira de Ensino de Biblioteconomia e Documentação. URL:< http: //www. abecin. org. br>

Barber, E. (1998). La formación profesional em bibliotecología y ciencia de la información en los países del Mercosur. Porto Alegre: Associação Brasileira de Ensino de Biblioteconomia e Documentação, 1998.

Barber, E. (2001). Informe sobre el Encuentro de Educación e Investigación en Bibliotecología, Archivología, Ciencia de la Información y Documentación de Iberoamérica y el Caribe (Granada, 21-25 de Febrero de 2000). // Cuarto Encuentro de Directores de Escuelas de Bibliotecología y Ciencia de la Información del Mercosur y Tercer Encuentro de Docentes de Escuelas de Bibliotecología y Ciencia

Scire. $10: 1$ (en.-jun. 2004) 145-157. 
de la Información del Mercosur. Montevidéu, 24-27 Maio 2000. [s. n. t.]. 6 p. (copia impresa).

Encontro de Diretores de Biblioteconomiae Ciência da Informação do Mercosul, 6 / Encontro de Docentes de Biblioteconomia e Ciência_da_Informaçãa do Mercosul, 5. Londrina, 22-25 Oct. 2002. Actas... URL: http://www.abecin.org.brp.

Encontro de Dirigentes de Cursos Superiores em Biblioteconomia do Mercosul. Porto Alegre, 28-28 set. 1996. A formação profissional em Biblioteconomia no Mercosul. Porto Alegre: Associação Brasileira de Ensino de Biblioteconomia e Documentação, 1996. $3 \mathrm{v}$.

Encuentro de Directores de los Cursos Superiores de Bibliotecología del Mercosur, 3; Encuentro de Docentes de Bibliotecología y Ciencia de la Información del Mercosur, 2, Santiago (Chile), 29-31 Oct. 1998. Formación de recursos humanos en el área de información en el Mercosur: compatibilización curricular; competencias del profesional de la información en el Mercosur. Santiago (Chile): Universidad Tecnológica Metropolitana, 1999.

Encuentro de Directores de los Cursos Superiores de Bibliotecología del Mercosur, 2; Encuentro de Docentes de Bibliotecología y Ciencia de la Información del Mercosur, 1. Buenos Aires, 27-29 Nov. 1997. La formación profesional en Bibliotecología y Ciencia de la Información en el Mercosur: acuerdos y recomendaciones. Buenos Aires: Universidad de Buenos Aires, Facultad de Filosofía y Letras, Departamento de Bibliotecología y Documentación, 1997. 15 p.

Encuentro de Educadores e Investigadores de Bibliotecología, Archivología y Ciencia de la Información de Iberoamerica y el Caribe, EDIBCIC, 3 (San Juan - Porto Rico, Ago. 1996). Informe de la Comisión de Pregrado. San Juan: Universidad de Puerto Rico, 1996. 4 p.

Encuentro de Educadores e Investigadores de Bibliotecología, Archivología y Ciencia de la Información de Iberoamerica y el Caribe, EDIBCIC, 5 (Granada - Esapña, 21-24 Feb. 2000). La formación de profesionales e investigadores de la información para la sociedad del conocimiento: actas del [...]. Granada: Universidad de Granada, Facultad de Biblioteconomía y Documentación, 2000. 506 p.

Fernández, S. M.; Giunti, G. M. (1999). Planes de estudio de las Escuelas de Bibliotecología, Archivología y Museología de Iberoamerica. Buenos Aires: Sociedad de Investigaciones Bibliotecológicas: ALP / IFLA, 1999.

Fernández, S. M. La ensenãnza de la Bibliotecología en la Faculdad de Filosofia y Letras. // Bibliotecología y Documentacion. 2 (1979) 5-30.

Guimarães, J. A. C. (2000). Estudos curriculares em Biblioteconomia no Mercosul: reflexões sobre uma trajetória. // Valentim, M. L. P. (ed.). Formação do profissional da informação. São Paulo: Polis, 2002. 49-88.

Guimarães, J. A. C.; Menezes, P. J.; Danuelo, J. C. (2003). Ensino de tratamento temático da informação nos cursos de Biblioteconomia do Mercosul: uma análise de capacitação de produção científica docente como subsídio ao delineamento de políticas integradas para a área. Marília, 2003. 141 f. Informe de investigación.

Scire. $10: 1$ (en.-jun. 2004) 145-157. 
Guimarães, J. A. C.; Rodrigues, M. E. F. (2003). A dimensão pedagógica da pesquisa nos cursos de Biblioteconomia do Mercosul: reflexões sobre uma trajectória de harmonização curricular. // Cadernos BAD. 1 (2003) 52-73.

Lampert, E. (1998). Educação e Mercosul: desafios e perspectivas. // Revista da Faculdade de Educação. 24:2 (jul/dez, 1998). URL: <http://www. scielo. br>.

Morosini, M. (1995). Harmonização curricular: necessidade emergente ou futura. // Anais do Primeiro Encontro Nacional de Entidades de Classe, Gramado (Brasil) 1995. Gramado: [s. n. ], 1995. 38-51.

Morosini, M. C. (1997). Internacionalização de sistemas universitários: o Mercosul. // Sguissardi, V.; Silva Junior, J. dos R. (Org). Políticas Públicas para a educação superior. Piracicaba: UNIMEP, 1997. 225-254.

Morosini, M. C. (1998). Universidade no Mercosul. 2. ed. São Paulo: Cortez, 1998.

Oliveira, E. F. T. (2001). A formação do Bibliotecário no âmbito do Mercosul: a realidade e as tendências no limiar do século XXI.. Marilia: Universidade Estadual Paulista, Faculdade de Filosofia e Ciências,. 2001. Tese Doutorado em Educação.

Santos, J. P. (1997). O ensino de Biblioteconomia no Mercosul: proposta de integração e harmonização curricular. São Paulo: Associação Paulista de Bibliotecários, 1997.

Santos, J. P. , Neves, I. C. B. (1998). Harmonização curricular em Biblioteconomia no Mercosul. Porto Alegre : ABEBD, 1998.

Valentim, M. L. P. (2000). Profissional da informação: formação, perfil e atuação profissional. São Paulo: Polis, 2000. 